\title{
Intermediate vision and other visual function parameters in patients after uneventful cataract surgery with implantation of diffractive intraocular lenses using the blending procedure
}

\author{
Karolina Podborączyńska-Jodko, Wojciech Lubiński, Michał Post \\ $2^{\text {nd }}$ Chair and Department of Ophthalmology, Pomeranian Medical University in Szczecin, Poland
}

\begin{abstract}
Aim of the study: To evaluate the intermediate vision and other visual function parameters after cataract surgery with blending implantation of AcrySof IQ ReSTOR intraocular lenses (IOLs) 3 months follow-up.

Material and methods: Forty eyes of 20 patients undergoing uneventful cataract surgery with blending implantation of the AcrySof IQ ReSTOR IOL (with addition +2.5 diopters (D) in dominant eye and $+3.0 \mathrm{D}$ in the fellow eye). Three months postoperatively the following factors were analyzed: binocular uncorrected intermediate visual acuity (UIVA), binocular uncorrected distance visual acuity (UDVA), binocular uncorrected near visual acuity (UNVA, logMAR scale), defocus curve, uncorrected binocular photopic and mesopic distance and photopic near contrast sensitivities, spectacle independence, subjective symptoms, patient
\end{abstract}

satisfaction (modified Visual Function Questionnaire - VFQ-25) and complications.

Results: Three months after binocular uneventful surgery UIVA from $60,70,80 \mathrm{~cm}$ were equal to $\log$ MAR $0.08,0.18,0.31$, respectively. UDVA was equal to logMAR 0.1 or better in all cases. All patients had binocular UNVA at $40 \mathrm{~cm}$ better than logMAR 0.3 . Contrast sensitivities in scotopic and photopic conditions were within normal age-matched limits. All patients were spectacle independent. A low level of halo perception less than 2 (0-4 scale) was reported. Mean overall patient satisfaction was very high 1.50 (1-5 scale). No postoperative complications were observed. Conclusions: Blending Restor lenses implantation was a safe and effective method for patients who want to be spectacle independent at intermediate vision without sacrificing distant and near vision. KEY WORDS: cataract surgery, blending procedure, diffractive lenses.

\section{INTRODUCTION}

Nowadays, about $60 \%$ of patients over 60 work with computers, smartphones and tablets, which is why good intermediate vision is very important for them [1].

Recently, for patients who want to be spectacle independent also for intermediate vision premium intraocular lenses (IOLs) such as trifocal or extended depth-of-focus (EDOF) IOLs have been available $[2,3]$. In the past it was also possible to obtain good results for intermediate vision using the blended bifocal IOL procedure. An example of these lenses is the Restor IOL, which was implanted in the Second Department of Ophthalmology in 2015. Bifocal diffractive IOLs give a possibility to see very well for two distances: far and near. However, intermediate vision in this type of lenses is also possible, which results from the depth of focus phenomenon [4]. Range of depth of focus is related to the plasticity of the brain cortex, which is different in a healthy population.
That is why the intermediate vision with bifocal IOLs is not perfect. Blended vision can be achieved by implantation in the dominant eye of an IOL with target refraction for distant vision, and in the fellow eye an IOL with target refraction for slight myopia (from -0.25 to -0.75 diopters (D)) or implantation of IOLs with different addition for near vision (commonly with lower addition in the dominant eye) [5]. In the literature, there is evidence that blending IOLs can improve binocular intermediate vision without compromising visual acuity for far distances [6]. Only a few study results describe the usefulness of blending Restor IOLs for improving intermediate vision; nevertheless the available results are quite good $[7,8]$. Therefore, we decided to analyze in our series of patients visual function results, especially intermediate vision after implantation of the Restor lenses with different addition: $+2.5 \mathrm{D}$ in the dominant eye and $+3.0 \mathrm{D}$ in the non-dominant eye (commonly referred to as the "mixed" ap- 
proach) and compare the results with those described in the literature after implantation of the trifocals IOL and EDOF IOLs.

\section{MATERIAL AND METHODS}

\section{Patients}

This retrospective case series included 40 eyes of 20 patients undergoing uneventful cataract surgery with implantation of the AcrySof ReSTOR IOL (Alcon) in 2015. Inclusion criteria were: significant cataract in patient older than 18 years and preoperative corneal astigmatism less than 0.75 D. Patients were excluded from the study when the following conditions were detected: ocular pathology other than cataract, systemic diseases with known influence on the retinal function. The study adhered to the tenets of the Declaration of Helsinki and was approved by the local Ethics Committee. Each patient was required to sign a consent form before participation in this study.

\section{Preoperative and postoperative evaluation}

Before the surgery, all patients underwent a complete ophthalmological examination. Classification of cataract

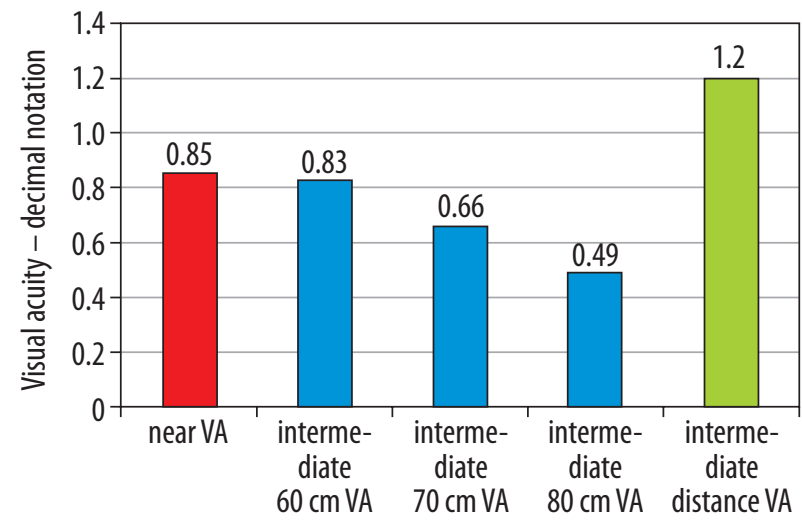

Figure 1. Mean binocular uncorrected near (red color), intermediate $(60,70$, $80 \mathrm{~cm}$ - blue color), distance (green color) visual acuity

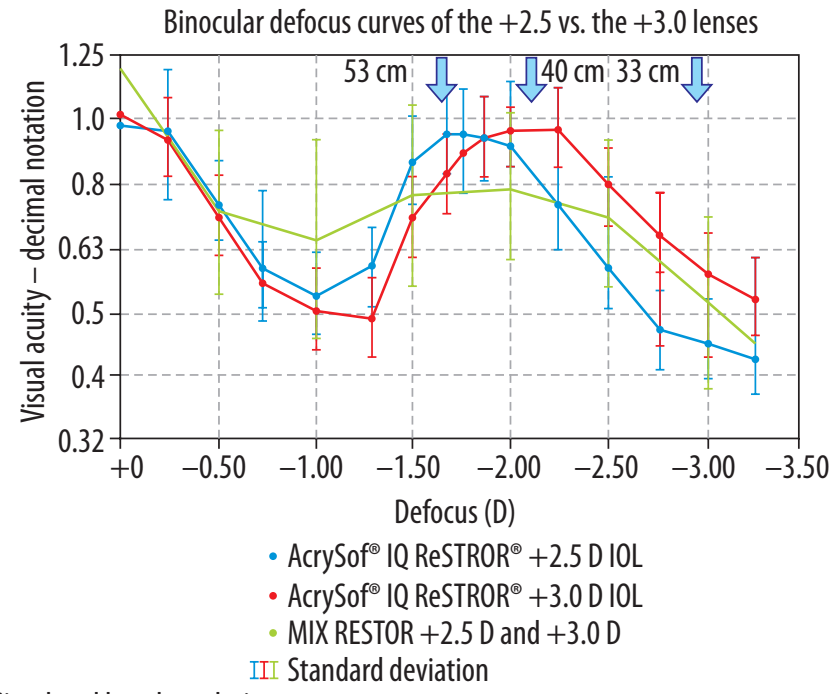

Simulated bench analysis.

Figure 2. Defocus curve was performed using Lens Opacities Classification System (LOCS) III. Biometry, keratometry and IOL power calculation were performed using the IOL Master partial coherence interferometry device (Zeiss - Meditec, Jena, Germany; software version 2005 AG). Target refraction was emmetropia; A - constant, as recommended by the manufacturer with SRK-T formula.

Three months after phacoemulsification in the second eye, the following parameters were evaluated: binocular uncorrected intermediate visual acuity (UIVA) [ $\log$ MAR chart $60,70,80 \mathrm{~cm}$ ], binocular uncorrected distance visual acuity (UDVA) [logMAR - ETDRS chart - $4 \mathrm{~m}$ ], binocular uncorrected near visual acuity (UNVA) [logMAR chart $-40 \mathrm{~cm}$ ], defocus curve, spectacle independence, binocular photopic $\left(85 \mathrm{~cd} / \mathrm{m}^{2}\right)$, mesopic $\left(4 \mathrm{~cd} / \mathrm{m}^{2}\right)$ for distance $(2.5 \mathrm{~m})$, binocular photopic $\left(85 \mathrm{~cd} / \mathrm{m}^{2}\right)$ for near $(35 \mathrm{~cm})$ uncorrected contrast sensitivities (CS; 1.5, 3, 6, 12, 18 c/deg, CSV-1000) and postoperative complications. We examined subjective symptoms (glare, halo) and the patient satisfaction modified Visual Function Questionnaire (VFQ-25) described by Leyland et al. [9].

\section{Surgical technique}

Under topical anesthesia, the same surgeon (W.L.) performed standard ultrasound phacoemulsification (phacochop procedure) with implantation of an IOL in-the-capsular-bag through a $2.2 \mathrm{~mm}$ clear corneal temporal incision.

\section{Data analysis}

Statistical analysis of the results was performed using Statistica Software using the Wilcoxon test with the significance level $p<0.05$.

\section{RESULTS}

Forty eyes of 20 patients were enrolled in the study with mean age of $67.89 \pm 7.09$ years. The most frequent types of cataract according to the LOCS III classification were NO3 and NC3.

\section{Visual and refractive outcomes}

Mean binocular uncorrected intermediate visual acuity values were $\log$ MAR 0.08 for $60 \mathrm{~cm}, \log$ MAR 0.18 for $70 \mathrm{~cm}$ and $\log$ MAR 0.31 for $80 \mathrm{~cm}$ (Figure 1 blue color). Mean binocular uncorrected distance visual acuity was logMAR -0.08 (Figure 1 green color). Mean binocular uncorrected near visual acuity was logMAR 0.07 (Figure 1 red color). The defocus curve is presented in Figure 2. All patients were totally spectacle independent. Contrast sensitivities in scotopic and photopic conditions were within normal agematched limits (Figures 3-5).

Patient satisfaction and visual disturbances are presented in Table I. A severe level of 'glare/halo' was detected in 25\% of patients.

\section{Complications}

No intra- or postoperative complications were observed. 


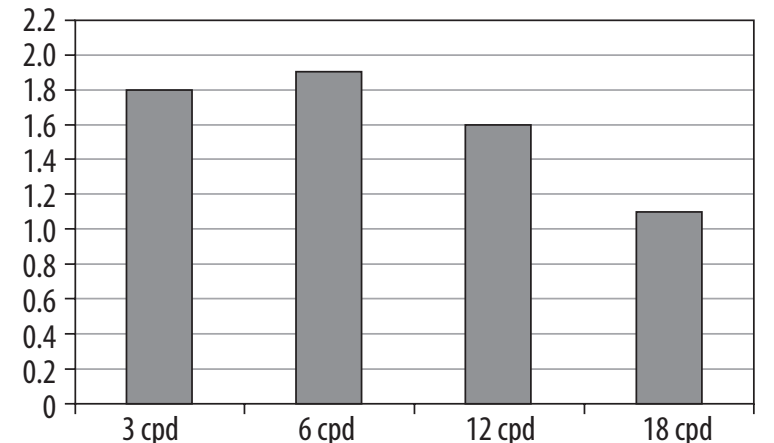

Figure 3. CSV 1000 - mean binocular photopic distance contrast sensitivity 3 months after surgery

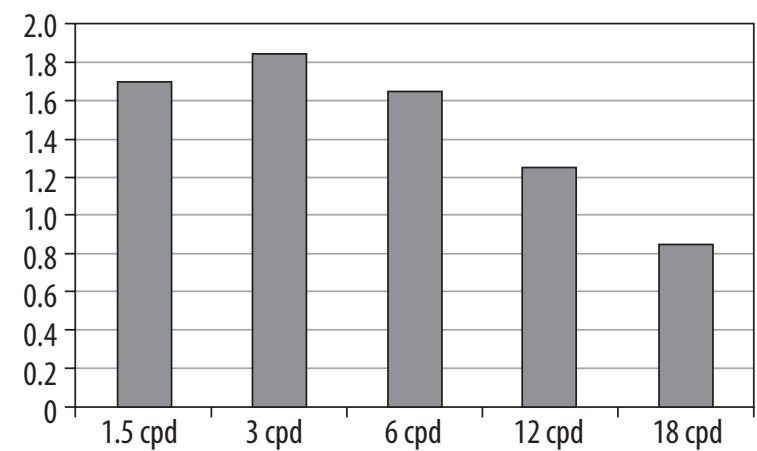

Figure 5. F.A.C.T. - mean binocular photopic near contrast sensitivity 3 months after surgery

\section{DISCUSSION}

The results of our study strongly support mixing the multifocal IOLs with different addition for near improved range of intermediate vision without compromising the distance and near vision.

Our patients achieved very good results for intermediate distance for $60 \mathrm{~cm}$ (mean visual acuity (VA) $0.08 \log \mathrm{MAR}$ ) and these results were comparable with those presented by Pedrotti et al. [8]. Mastropasqua et al. described for the same distance VA $0.06 \log$ MAR. Values of intermediate VA obtained in our study were better, compared to the results achieved after implanting in both eyes lenses with $+3.0 \mathrm{D}$ addition (VA mean range: from $0.138 \log M A R$ to 0.41 ) and with lenses with addition of $+2.5 \mathrm{D}$ (VA mean logMAR 0.44 ) $[7,10,11]$. Intermediate vision for $60 \mathrm{~cm}$ when mixing IOLs was comparable to binocular trifocal IOL implantations and with EDOF lenses (VA range: from logMAR 0.0 to $\log M A R$ $0.11)$ [12-14].

In our study we also analyzed intermediate vision for 70 and $80 \mathrm{~cm}$ and the obtained results were very good and equal to 0.18 and $0.31 \log M A R$, respectively. In the literature, the results for binocular VA from $70 \mathrm{~cm}$ were as follows: $0.24 \log \mathrm{MAR}$ with addition $+2.5 \mathrm{D}$ and $0.53 \log \mathrm{MAR}$ with addition $+3.0 \mathrm{re}$ spectively. VA for $70 \mathrm{~cm}$ achieved in our study was comparable to VA published by Pedrotti when binocular implantation with addition $+2.5 \mathrm{D}$ was used, but worse when lenses with addition +3.0 D were implanted in both eyes [8].

In our study VA for $80 \mathrm{~cm}$ was worse (0.31 logMAR) than VA with trifocal IOLs (mean 0.09 $\log$ MAR) [15]. Comparable

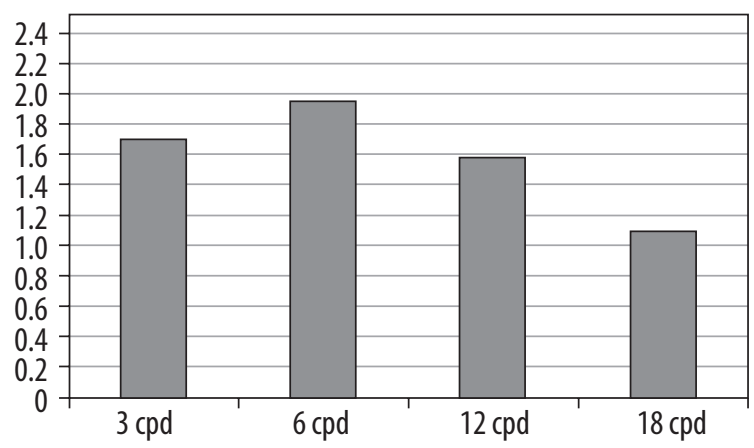

Figure 4. CSV 1000 - mean binocular mesopic distance contrast sensitivity 3 months after surgery

Table I. Patients' satisfaction test - modified VFQ-25 questionnaire (binocular, unaided vision)

\begin{tabular}{|l|c|c|c|}
\hline Activities & $\begin{array}{l}\text { Best } \\
\text { score }\end{array}$ & $\begin{array}{l}\text { Worst } \\
\text { score }\end{array}$ & $\begin{array}{l}\text { Mean outcome } \\
\text { after 3 months }\end{array}$ \\
\hline Reading newspapers & 1 & 5 & 1.55 \\
\hline Using computer & 1 & 5 & 1.19 \\
\hline Seeing road signs & 1 & 5 & 1.00 \\
\hline Daytime driving & 1 & 5 & 1.00 \\
\hline Night driving & 1 & 5 & 1.00 \\
\hline Difficult-situation driving & 1 & 5 & 1.00 \\
\hline Seeing up close & 1 & 5 & 1.65 \\
\hline Steps and stairs & 1 & 5 & 1.07 \\
\hline General visual acuity quality & 1 & 5 & 1.50 \\
\hline Worry about visual acuity & 1 & 5 & 1.80 \\
\hline $\begin{array}{l}\text { Accomplish less due } \\
\text { to visual acuity }\end{array}$ & 5 & 1 & 4.85 \\
\hline Frustrated due to visual acuity & 5 & 1 & 4.90 \\
\hline Level of 'glare/halo' & 0 & 4 & 0.60 \\
\hline
\end{tabular}

analysis of intermediate vision suggested that this vision for longer distances is better for trifocal IOLs.

Our patients also achieved satisfactory VA results for near for $40 \mathrm{~cm}$ (mean VA $0.07 \log$ MAR) and it was similar to those presented by other authors using the blending technique (mean VA $0.10 \log$ MAR). From other study results it is known that the patients with implantation of IOLs with addition $+2.5 \mathrm{D}$ in both eyes achieved $0.19 \log \mathrm{MAR}$, but after implantation with addition $+3.0 \mathrm{D}$ they achieved $0.36 \log \mathrm{MAR}$ [8]. Binocular visual acuity for near after implantation of trifocal IOL (AT LISA tri, Physiol Fine Vision, Tecnis Symfony) ranged between logMAR 0.0 and 0.04 and was comparable to our results but better in patients after implantations of lenses with the same addition in both eyes $[16,17]$.

The analysis of the binocular defocus curve indicated that the range of good intermediate vision after implantation of lenses with different addition was wider than the range after the implantation of the lenses with the addition $+2.5 \mathrm{D},+3.0 \mathrm{D}$. Comparison of defocus curve results with the blending procedure in our study to the defocus curve results 
described by Hamid indicated that vision between 80 and $70 \mathrm{~cm}$ (defocus $-1.0 \mathrm{D}$ to $-1.5 \mathrm{D}$ ) was better in the trifocal group [18].

The study published by Gill et al. proved that only EDOF IOL showed the profile with a single extended focus opposite to bifocal and trifocal lenses [19].

In our study, mean binocular distance VA was equal to $-0.08 \log M A R$ and it was comparable to the data described by Mastropasqua using the same blending technique. In a group of trifocal IOLs the mean distance VA ranged between -0.05 to -0.1 for AT Lisa Tri lenses and about 0.0 logMAR for Physiol Fine Vision lenses and for Symfony [8, 20-22]. In summary, the binocular distance VA after blended implantation of IOLs with addition $+2.5 \mathrm{D},+3.0 \mathrm{D}$, trifocal or EDOF lenses was very good and allowed comparable results to be achieved.

Our patients were totally spectacle independent. It seems that the concept of blending lenses is a good option for patients who do not want to use glasses in their active life. Total spectacle independence was not observed in patients with bilateral implantation of the diffractive AcrySof IQ Restor SN6AD1 with addition +3.0 D (78\% of patients spectaclefree). In another study about $80-100 \%$ of patients after implantation of trifocal IOLs were spectacle-free [15]. Law et al. reported that a limited percentage of patients implanted with the same IOL (addition $+2.5 \mathrm{D}$ ) had some difficulties to perform near and intermediate visual tasks without glasses, such as reading the newspaper or working with the computer [23]. Kohnen et al. found in their study with the AT LISA trifocal IOL that $100 \%$ of the patients were independent of spectacles for distant and intermediate vision, but $12 \%$ of patients needed occasionally near correction. In a study with the FineVision IOL $80 \%$ of the patients were reported to be completely spectacle independent [24]. The spectacle independence with the Symfony lenses was also very high - the mean was $90 \%$. One of the possible explanations of the total spectacle independence found in our study is very precise selection of patients and also the small number of patients included in the study group.

In our study a high level of CS for distance and near was observed even with high spatial frequencies. This fact requires further tests to explain the reason. It is difficult to compare the results obtained by other authors, since they used different lighting conditions and not the same equipment [25]. In our study general patient satisfaction was very high and it was in agreement with the results obtained using implantation of lenses with different addition $+2.5 \mathrm{D},+3.0 \mathrm{D}$, blending, trifocal and EDOF lenses. The patients had no trouble with driving in day or night conditions and using smartphones [26]. It was also difficult to precisely compare obtained results of patient satisfaction with other study results because different tests were used by other authors. Major unwanted effects such as glare and halo were detected in our study in $25 \%$ of patients. Frequency of photopic phenomena after implantation of binocular lenses with the blending procedure was similar to that described by other authors using trifocal IOLs or after bilateral implantation with addition $+2.5 \mathrm{D},+3.0 \mathrm{D}$ and EDOF lenses [27]. In our patients detected unwanted effects were acceptable and no patient required explantation of the IOL for this reason. It is known from the literature that longer adaptation time significantly contributes to the disappearance of this photopic phenomena. The followup in our study group lasted only three months. It should be expected that after a longer period of time, the percentage of patients suffering from photopic phenomena as well as the level of its perception will be lower [28].

\section{CONCLUSIONS}

In conclusion, blended Restor lenses implantation was a safe and effective method for patients who want to be spectacle independent for intermediate distance without compromising distant and near vision.

Retrospective comparative analysis of visual outcomes, spectacle independence, and the frequency of unwanted effects after implantation of lenses with the blending procedure, trifocals and EDOF IOL implantation indicate that the results did not differ significantly, with the exception of worse intermediate vision for longer distances.

\section{DISCLOSURE}

The authors declare no conflict of interest.

\section{References}

1. Tang Z, Zhang H, Yan A, Qu C. Time is money: the decision making of smartphone high users in gain and loss intertemporal choice. Front Psychol 2017; 8: 363.

2. Pedrotti E, Bruni E, Bonacci $E$, et al. Comparative analysis of the clinical outcomes with a monofocal and an extended range of vision intraocular lens. J Refract Surg 2016; 32: 436-442.

3. Shen Z, Lin Y, Zhu Y, et al. Clinical comparison of patient outcomes following implantation of trifocal or bifocal intraocular lenses: a systematic review and meta-analysis. Sci Rep 2017; 7: 45337.

4. Yoon SY, Song IS, Kim JY, et al. Bilateral mix-and-match versus unilateral multifocal intraocular lens implantation: long-term comparison. J Cataract Refract Surg 2013; 39: 1682-1690.

5. Madrid-Costa D, Ruiz-Alcocer J, Ferrer-Blasco T, et al. Optical quality differences between three multifocal intraocular lenses: bifocal low add, bifocal moderate add, and trifocal. J Refract Surg 2013; 29: 749-754.

6. Jacobi FK, Kammann J, Jacobi KW, et al. Bilateral implantation of asymmetrical diffractive multifocal intraocular lenses. Arch Ophthalmol 1999; 117: 17-23.

7. Gundersen K. Potvin R. Comparative visual performance with monofocal and multifocal intraocular lenses. Clin Ophthalmol 2013; 7: 1979-1985.

8. Pedrotti E, Mastropasqua R, Passilongo M, et al. Comparison of two multifocal intraocular lens designs that differ only in near add. J Refract Surg 2014; 30: 754-760. 
9. Leyland MD, Langan L, Goolfee F, et al. Prospective randomized double-masked trial of bilateral multifocal, bofocal or monofocal intraocular lenses. Eye 2002; 16: 481-490.

10. Mastropasqua R, Pedrotti E, Passilongo $\mathrm{M}$, et al. Long-term visual function and patient satisfaction after bilateral implantation and combination of two similar multifocal IOLs. J Refract Surg 2015; 31: 308-314.

11. Hayashi K, Ogawa S, Manabe S, Hirata A. Visual outcomes in eyes with a distance-dominant diffractive multifocal intraocular lens with low near addition power. Br J Ophthalmol 2015; 99: 1466-1470.

12. Vryghem JC, Heireman S. Visual performance after the implantation of a new trifocal intraocular lens. Clin Ophthalmol 2013; 7: 1957-1965.

13. Torun A, Duman E, Simsek S. Clinical outcomes of a new diffractive trifocal intraocular lens with Enhanced Depth of Focus (EDOF). BMC Ophthalmol 2016; 16: 208.

14. Cochener B, Boutillier G, Lamard M, Auberger-Zagnoli C. A comparative evaluation of a new generation of diffractive trifocal and extended depth of focus intraocular lenses. J Refract Surg 2018; 34: 507-514.

15. Kohnen T, Titke C, Böhm M. Trifocal intraocular lens implantation to treat visual demands in various distances following lens removal. Am J Ophthalmol 2016; 161: 71-77.

16. Maxwell WA, Cionni RJ, Lehman RP, Modi SS. Functional outcomes after bilateral implantation of apodized power. Randomized multicenter clinical study. J Cataract Refract Surg 2009; 35: 2054-2061.

17. Lubiński W, Podborączyńska-Jodko K, Gronkowska-Serafin J, Karczewicz D. Visual outcomes three and six months after amplantation of diffractive and refractive multifocal IOL combination. Klin Oczna 2011; 113: 209-215.

18. Hamid A, Sokwala A. A more natural way of seeing: visual performance of three presbyopia correcting intraocular lenses. Open J Ophthalmol 2016; 6: 176-183.

19. Gil MA, Varon C, Cardona G, Buil JA. Visual acuity and defocus curves with six multifocal intraocular lenses. Int Ophthalmol 2020; 40:393-401.

20. Mendicute J, Kapp A, Lévy P, et al. Evaluation of visual outcomes and patient satisfaction after implantation of a diffractive trifocal intraocular lens. J Cataract Refract Surg 2016; 42: 203-210.

21. Madrid-Costa D, Ruiz-Alcocer J, Ferrer-Blasco T, et al. Optical quality differences between three multifocal intraocular lenses: bifocal low add, bifocal moderate add, and trifocal. J Refract Surg 2013; 29: 749-754.

22. Tomas-Juan J. Multifocal IOLs with apodized diffractive central zone and refractive periphery: optical performance and clinical outcomes. J Emmetropia 2014; 5: 155-166.

23. Law EM, Aggarwal RK, Kasaby H. Clinical outcomes with a new trifocal intraocular lens. Eur J Ophthalmology 2014; 24: 501-508.

24. Kretz FT, Choi CY, Müller M, et al. Visual outcomes, patient satisfaction and spectacle independence with a trifocal diffractive intraocular lens. Korean J Ophthalmol 2016; 30: 180-191.

25. Vingolo E, Carnevale C, Fragiotta S, et al. Visual outcomes and contrast sensitivity after bilateral implantation of multifocal intraocular lenses with +2.50 or +3.0 diopter addition: 12-month follow-up. Semin Ophthalmol 2017; 32: 588-592.

26. Rosen E, Alió $\mathrm{JL}$, Dick HB, et al. Efficacy and safety of multifocal intraocular lenses following cataract and refractive lens exchange: metaanalysis of peer-reviewed publications. J Cataract Refract Surg 2016; 42: 310-328.

27. Cochener B. Prospective clinical comparison of patient outcomes following implantation of trifocal or bifocal intraocular lenses. J Refract Surg 2016; 32: 146-151.

28. Chen T, Yu F, Lin H, Zhao Y. Objective and subjective visual quality after implantation of all optic zone diffractive multifocal intraocular lenses: a prospective, case-control observational study. Br J Ophthalmol 2016; 100: 1530-1535. 